\title{
Problems and solutions for the diagnosis of gestational diabetes mellitus in non metropolitan areas in India
}

\author{
V. Seshiah, N. Bhavatharini, Sanjeev V. K., Changanidi A. R. A., R. Aruyerchelvan*
}

\section{S. R. C. Diabetes Care Center, Erode, Tamil Nadu, India}

Received: 14 October 2019

Accepted: 30 January 2020

\section{*Correspondence:}

Dr. R. Aruyerchelvan,

E-mail: srcdiab@yahoo.com

Copyright: (C) the author(s), publisher and licensee Medip Academy. This is an open-access article distributed under the terms of the Creative Commons Attribution Non-Commercial License, which permits unrestricted non-commercial use, distribution, and reproduction in any medium, provided the original work is properly cited.

\begin{abstract}
Management of gestational diabetes mellitus and hyperglycemia in pregnancy is challenging in developing country like India. There are several logistic, socioeconomic and cultural issues faced by the care providers and the patients alike. In order to tackle these challenges, Diabetes in Pregnancy Study Group of India (DIPSI) recommendations consisting of a single glucose challenge test is ideal to evaluate the glucose intolerance and early initiation of doctors' prescription. This review article explores the practical challenges associated with managing gestational diabetes mellitus and recommendations to overcome these challenges in the public health system.
\end{abstract}

Keywords: Gestational diabetes mellitus, Hyperglycemia, Pregnancy, Primary health care

\section{INTRODUCTION}

Hyperglycaemia in pregnancy (HIP) is a condition wherein a woman during pregnancy is detected with slightly elevated blood glucose levels (gestational diabetes mellitus (GDM)) and also with previously known history of diabetes mellitus. ${ }^{1}$ It has been estimated that most (75-90\%) of cases of high blood glucose during pregnancy are gestational diabetes, which primarily affects pregnant women usually during the First, second and third trimesters of pregnancy. ${ }^{2}$ In some women diabetes may be diagnosed in the first trimester of pregnancy although in majority of such cases, diabetes more often could have existed before pregnancy, but was undiagnosed.

The vast majority $(88 \%)$ of cases of hyperglycaemia in pregnancy were in low and middle income countries (LMIC), where access to maternal care is often limited. The prevalence of hyperglycaemia in pregnancy, as a proportion of all pregnancies, increases rapidly with age and is highest in women over the age of 45 years $(45.4 \%)$, although there are fewer pregnancies in that age group. Due to higher fertility rates in younger women, nearly half $(48.9 \%)$ of all cases of hyperglycaemia in pregnancy (10.4 million) occurred in women under the age of 30 . Prevalence of GDM in India is 27.9 as per IDF 2017 estimate in age group 20-49 years. $^{3}$

GDM arises because there is relative insulin deficiency (insulin resistance) due to hormone production by the placenta. ${ }^{4}$ Other risk factors for GDM include older EG (modified from Lakatta) age, overweight or obesity, excessive weight gain during pregnancy, a family history of diabetes and a history of stillbirth or giving birth to an infant with a congenital abnormality. GDM usually exists as a transient disorder during pregnancy and resolves once the pregnancy ends. However, pregnant women with hyperglycaemia are at higher risk of developing GDM in subsequent pregnancies and about half of women with a history of GDM will develop type II Diabetes within five to ten years after delivery. GDM also results in various complications in the babies including a higher lifetime risk of obesity, type 2 diabetes and other non- 
communicable diseases (NCD). In addition, the adverse pregnancy outcomes including high blood pressure and a large baby for gestational age, foetal macrosomia also ensue. ${ }^{5}$ Identification of hyperglycaemia in pregnancy combined with good control of blood glucose during pregnancy can reduce these risks. Simple initiatives including healthy diet, gentle exercise and blood glucose monitoring coupled with insulin therapy are the mainstay in management of hyperglycemia in pregnancy.

In a country like India, the population of GDM is diverse, spread across the urban, sub-urban and rural areas. There are several challenges faced by the rural and sub-urban population in accessing optimal health care service. This review explores the various challenges and issues pertaining to GDM management in the rural and suburban areas of India.

\section{SCENARIO OF GDM IN NON-METRO CITIES IN INDIA}

In a recent survey done in 10 non metro government hospitals, it was observed that over $90 \%$ of the antenatal mothers are unable to visit the center in fasting status. Our primary health centres (PHC) run Antenatal clinics (ANC) on every Tuesdays, weekly once. When the patients are required to visit the $\mathrm{PHC}$ in fasting status for a glucose tolerance test (GTT), there is undue delay owing to certain factors, because of which this results in re-visit of the GDM patients during their turn in the next week, thereby impacting the quality of care. In such scenarios, there is an increased likelihood of overcrowding and there is further delay in diagnosis if she happens to be GDM.

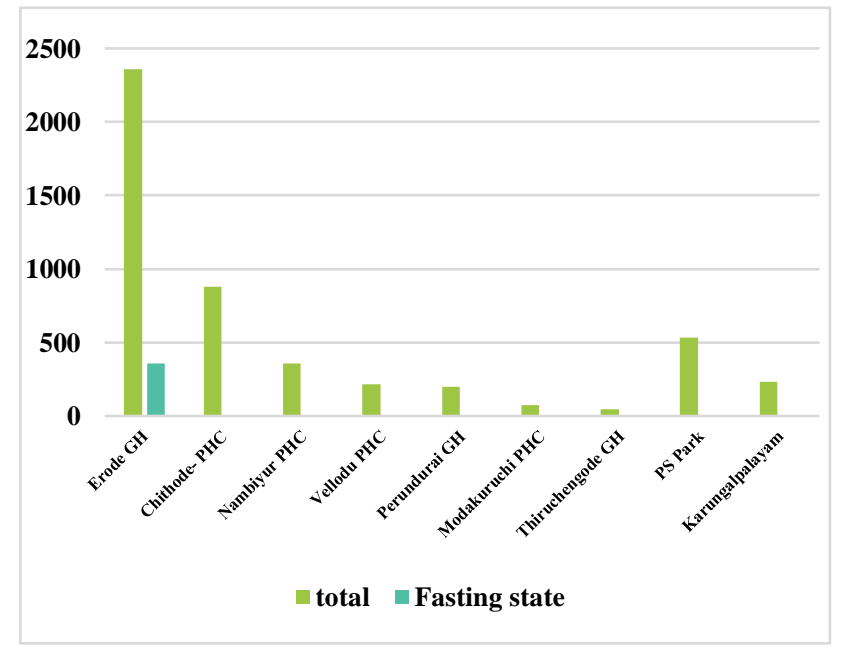

Figure 1: Proportion of pregnant women attending antenatal clinic in fasting state in southern districts of Tamil Nadu.

In certain remote locations, the lack of adequate stock for GTT testing, including strips, reagents, syringes, etc hampers with timely testing for the GDM patients. Moreover, in certain centres, non-availability of the paramedical personnel has also been observed. These inadequacies result in drop outs we miss many GDM in this drop outs which is rampantly prevalent in the public health infrastructure.

As per various recommendations, two visits are required for the antenatal evaluation of GDM status. This is practically draining on various resources for both the patients and for the care providers. A considerable enhancement on the care provider's skills will be grateful in minimizing this work load and will ease out various issues at all levels.

\section{CHALLENGES ASSOCIATED WITH FASTING DURING ANTENATAL CHECK-UPS}

Time factor is one of the key reasons which hinder with fasting. The centres begin only by $8 \mathrm{AM}$, for each antenatal patient to get her turn takes another 1 hour. However, it is sometimes impractical for an antenatal woman to remain fasting till that time. Although to give blood sugar in fasting consuming glucose for GTT takes less than five minutes, for each case the over crowd in gat that point of time further delays the fasting period. By the time about 100-150 patients are finished with blood sugar in fasting and given glucose load, a few would have crossed their 1 hour and some 2 hour also.

All women are committed to their families and care of their family members including domestic animals is one of their utmost priorities. In such situations, traditionally woven rural and sub-urban societies continue to resist placing their health as a priority. therefore, they are often unable to report to the laboratory in fasting status before completion of their household chores and responsibilities due to longer time.

Certain health centers are situated in remote locations with minimal access through public transport. The frequency of bus is not much and they have to walk to get the next bus. It takes quite a distance minimum of $1 / 2 \mathrm{~km}$ to reach the bus stop. Women are generally dependent on spouses or relatives for the AN visit, which reduces their compliance for fasting as the timings have to be based on the availability of transport systems. More so, if they are daily wage earners, two wages are lost on a single visit.

In some situations, considering the distance, women pool their transport to the health centers. In such scenarios, they tend to crowd the facility and this inherently prolongs their fasting duration.

Above all, the traditional Indian society strongly believes that pregnant women should not fast for a long time and is against fasting during antenatal period. This cultural taboo results in poor compliance towards fasting.

If they are asked to come next week for blood samples and there is undue delay due to various constraints, there is a colossal damage caused to their livelihood as they 
tend to lose their day's wages which severely demotivates them to fasting blood sugar evaluation because they have to come again next week for fasting.

The fasting value in any pregnant women is lower due to heightened insulin sensitivity as muscle takes up the glucose and neoglucogenesis comes down.

Moreover, these pregnant women are prone to ketosis due to accelerated starvation. Fetus is a continuously eating parasite in an intermittently eating host.

With a majority of the antenatal women suffering from hyperemesis, hyperosmolarity associated with GTT solution leads to GI intolerance in fasting, aggravating nausea and vomiting. They tolerate the glucose well in non-fasting state.

Continuous power supply and availability of drinking water is yet another problem. The health care providers are much confused with whom to test, when to test and how to test. GTT is time consuming process with cumbersome values and has to be done in fasting state according to recommendations.

\section{RECOMMENDATIONS AND SOLUTIONS}

Effective prevention of complications of GDM is possible by early detection and appropriate treatment. It is a known fact that Indians are 11 times more prone to GDM due to various factors including genetic, lifestyle, family history, late marriages, late childbirth etc., world health organization (WHO) on its report on observations and recommendations (2014) observed that, for a pregnant woman, the request to attend fasting for blood test may not be realistic because of the long travel to the clinic in many parts of the world, and increased tendency to nausea in the fasting state. Consequently, non fasting testing may be the only practical option. As per our national guidelines, the DIPSI criteria of glucose challenge test (GCT) can be carried out either in fasting or fed state, thereby fulfilling FIGO, WHO and WDF recommendations. A two-hour value after $75 \mathrm{~g}$ of OGTT in fasting or non fasting state, with a value $>140 \mathrm{mg} / \mathrm{dl}$ may be diagnostic of GDM. ${ }^{6}$ This one step procedure solves a variety of challenges associated with repeated visits to the health facility, problems associated with fasting, etc. A well calibrated glucometer and assured supply of power complimented with adequate and periodic monitoring of stock and supplies are the bare minimum requirements to fulfil these recommendations. Several studies done to evaluate the feedback have shown better compliance with these recommendations and are a welcome relief for both care providers and patients. The other practical advantages include least disturbance to the mother's routine activities. In addition, this test serves as both screening and diagnostic procedure and universal testing is possible.
One test- 75 grams glucose in fasting or non fasting state, one value- to diagnose 2 hours $\geq 140 \mathrm{mg} / \mathrm{dl}$ and one target- for monitoring: 2 post prandial glucose <120 $\mathrm{mg} / \mathrm{dl}$.

\section{CONCLUSION}

Management of GDM and HIP is challenging in developing country like India, wherein the population is large and diverse. There are several issues faced by the care providers and the patients alike in the rural and suburban areas, associated with various other non-medical factors. In order to tackle these challenges, DIPSI recommendations consisting of a single GCT is ideal to evaluate the glucose intolerance and early initiation of doctors' prescription. This easy economical single step procedure may be implemented as a routine protocol in all the public health centres for better and effective diabetes management in pregnancy.

Funding: No funding sources Conflict of interest: None declared Ethical approval: Not required

\section{REFERENCES}

1. Hod M, Kapur A, Sacks DA, Hader E, Agarwal M, Di Renzo GC, et al. The International Federation of Gynaecology and Obstetrics (FIGO) Initiative on gestational diabetes mellitus: A pragmatic guide for diagnosis, management, and care. Intl J Gynaecol Obstet. 2015;131(3):173-221.

2. Guariguata L, Linnenkamp U, Beagley J, Whiting DR, Cho NH. Global estimates of the prevalence of hyperglycaemia in pregnancy. Diabetes Res Clin Pract. 2014;103(2):176-85.

3. International Diabetes Federation IDF Diabetes Atlas. 8th edition; 2017.

4. World Health Organization. Diagnostic criteria and classification of hyperglycaemia first detected in pregnancy. World Health Organization; 2013.

5. Fetita LS, Sobngwi E, Serradas P, Calvo F, Gautier JF. Consequences of fetal exposure to maternal diabetes in offspring. J Clin Endocrinol Metab. 2006;91(10):3718-24.

6. Tripathi R, Verma D, Gupta VK, Tyagi S, Kalaivani $\mathrm{M}$, Ramji S, et al. Evaluation of $75 \mathrm{~g}$ glucose load in non fasting state [Diabetes in Pregnancy Study Group of India (DIPSI) criteria] as a diagnostic test for gestational diabetes mellitus. Indian J Med Res. 2017;145(2):209-14.

Cite this article as: Seshiah V, Bhavatharini N, Sanjeev VK, Changanidi ARA, Aruyerchelvan R. Problems and solutions for the diagnosis of gestational diabetes mellitus in non metropolitan areas in India. Int $\mathbf{J}$ Community Med Public Health 2020;7:1607-9. 